\title{
Enabling structure-based drug design of Tyk2 through co-crystallization with a stabilizing aminoindazole inhibitor
}

Maria A Argiriadi ${ }^{{ }^{*}{ }^{+}}$, Eric R Goedken ${ }^{1 \dagger}$, David Banach ${ }^{1}$, David W Borhani ${ }^{2}$, Andrew Burchat ${ }^{3}$, Richard W Dixon ${ }^{4}$, Doug Marcotte ${ }^{5}$, Gary Overmeyer ${ }^{6}$, Valerie Pivorunas ${ }^{7}$, Ramkrishna Sadhukhan ${ }^{6}$, Silvino Sousa ${ }^{6}$, Nigel St John Moore ${ }^{2}$, Medha Tomlinson ${ }^{6}$, Jeffrey Voss ${ }^{1}$, Lu Wang $^{3}$, Neil Wishart ${ }^{3}$, Kevin Woller ${ }^{8}$ and Robert $\vee$ Talanian $^{1}$

\begin{abstract}
Background: Structure-based drug design (SBDD) can accelerate inhibitor lead design and optimization, and efficient methods including protein purification, characterization, crystallization, and high-resolution diffraction are all needed for rapid, iterative structure determination. Janus kinases are important targets that are amenable to structure-based drug design. Here we present the first mouse Tyk2 crystal structures, which are complexed to 3-aminoindazole compounds.

Results: A comprehensive construct design effort included $\mathrm{N}$ - and C-terminal variations, kinase-inactive mutations, and multiple species orthologs. High-throughput cloning and expression methods were coupled with an abbreviated purification protocol to optimize protein solubility and stability. In total, 50 Tyk2 constructs were generated. Many displayed poor expression, inadequate solubility, or incomplete affinity tag processing. One kinase-inactive murine Tyk2 construct, complexed with an ATP-competitive 3-aminoindazole inhibitor, provided crystals that diffracted to $2.5-2.6 \AA$ resolution. This structure revealed initial "hot-spot" regions for SBDD, and provided a robust platform for ligand soaking experiments. Compared to previously reported human Tyk2 inhibitor crystal structures (Chrencik et al. (2010) J Mol Biol 400:413), our structures revealed a key difference in the glycine-rich loop conformation that is induced by the inhibitor. Ligand binding also conferred resistance to proteolytic degradation by thermolysin. As crystals could not be obtained with the unliganded enzyme, this enhanced stability is likely important for successful crystallization and inhibitor soaking methods.

Conclusions: Practical criteria for construct performance and prioritization, the optimization of purification protocols to enhance protein yields and stability, and use of high-throughput construct exploration enable structure determination methods early in the drug discovery process. Additionally, specific ligands stabilize Tyk2 protein and may thereby enable crystallization.
\end{abstract}

Keywords: Tyk2, Jak kinase, Crystallization, Proteolysis

\section{Background}

Janus kinases (Jaks) have broad roles in immune regulation via their action in cytokine signalling [1-3]. These non-receptor tyrosine kinases phosphorylate receptor

\footnotetext{
* Correspondence: maria.argiriadi@abbott.com

tEqual contributors

'Department of Molecular \& Cellular Pharmacology, Abbott Laboratories, Worcester, MA, USA

Full list of author information is available at the end of the article
}

chains, which in turn recruit and phosphorylate members of the Signal Transducer and Activator of Transcription (STAT) family [2,4]. The Jak family comprises Jak1, Jak2, Jak3 and Tyk2. These enzymes have very similar domain structures, containing a FERM domain, an SH2 domain, a pseudokinase domain, and a catalytic tyrosine kinase domain. Jaks serve overlapping but distinct functions in cytokine signaling, as demonstrated by knockout, mutation and other studies [5-9].

\section{Biomed Central}


Because of their roles in the signaling of many important cytokines, hormones, and growth factors such as IL-2, IL-4, IL-6, IL-7, IL-12, IL-13, IFN- $\alpha$, IFN- $\gamma$, Epo, and GM-CSF $[10,11]$, Jak inhibitors might have wide application in the treatment of inflammatory, myeloproliferative and autoimmune diseases, and therefore the Jak enzymes are attractive targets for drug discovery. Initial studies with Jak3 inhibitors were aimed at preventing solid organ transplant rejection [12,13]. More recent studies have explored the potential of such compounds in chronic autoimmune diseases such as rheumatoid arthritis and psoriasis [14-16]. For example, tofacitinib (CP-690,550), which inhibits Jak1, Jak2, and Jak3, has demonstrated efficacy in Phase II trials for rheumatoid arthritis [17-19]. Ruxolitinib (Jakafi ${ }^{\circledR}$ ), a dual Jak1 and Jak2 inhibitor [20], was recently approved for the treatment of myelofibrosis, a disorder involving myeloproliferative neoplasm.

The development of Tyk2 inhibitors is less advanced. Tyk2 functions together with Jak2 in the signaling of IL-12 and IL-23 via its interaction with the IL-12R $\beta 1$ receptor chain, and in the coordinated phosphorylation of STAT3 \& STAT4 [4,21]. Human Tyk2 gene deficiency causes defects in signaling of multiple cytokines, including IL-6, IL-10, IL-12 and IL-23, and reduced production of IFN $\gamma$ [5]. Furthermore, Tyk2-deficient mice are resistant to experimental autoimmune encephalomyelitis, a model for multiple sclerosis [22,23]. Given the importance of Tyk2-dependent downstream cytokine signaling in this and other diseases such as rheumatoid arthritis and Crohn's disease, Tyk2 inhibitors have the potential to be important therapeutics.

Because Jak family active sites exhibit high sequence identity, designing inhibitors selective within the family is challenging. One way to approach this challenge is to target active site regions that differ in conformation between homologs. To identify these "hot-spot" regions, we set out to obtain multiple crystal structures of Tyk2 in complex with a variety of ligands representing diverse chemotypes. At the time of our initial work, only Jak2 and Jak3 crystal structures had been published [24,25]. Robust Tyk2 crystallography allowing for the soaking of multiple inhibitors, essential for rapid throughput in structure-based drug design, had not been described. After exploring multiple constructs, we obtained crystals of mouse Tyk2 in the presence of 3-aminoindazole inhibitors that diffracted to $2.5-2.6 \AA$ resolution. The inclusion of a ligand was absolutely required to obtain high-quality crystals, and we found through limited proteolysis experiments that the enzyme is significantly stabilized by binding to such ATP-competitive inhibitors. This process enabled the determination of multiple inhibitor-soaked Tyk2 crystal structures, forming the basis of an extensive SBDD program.

\section{Results and discussion}

\section{Construct design and purification strategies}

Several strategies were employed to obtain sufficient protein purification yields for crystallization: (1) variation of $\mathrm{N}$ - and $\mathrm{C}$-terminal boundaries of the Tyk2 catalytic domain (some constructs included the pseudokinase domain); (2) variation of the affinity purification tag; (3) introduction of a kinase-inactivating mutation; and (4) use of multiple orthologs. Table 1 lists the different strategies and examples employed for Tyk 2 construct design. After exploring roughly 40 constructs (list shown in Additional file 1 Table 1), we prioritized a mouse construct that produced adequate amounts of soluble protein for crystallization (GST-Tev-muTyk2 (870-1170) Asp1016Ala). The human and mouse Tyk2 catalytic domain sequences are highly conserved (91\% identity in catalytic domain, 78\% identity overall); however, several divergent surface residues had the potential to impact protein aggregation and crystallization behavior. A glutathione-S-transferase (GST) tag was included to increase solubility during early stages of purification, and the Asp1016Ala kinase-inactive mutation was introduced to increase conformational homogeneity by preventing multiple phosphorylation states; this mutation also increased expression approximately threefold (data not shown). Asp1016 is the conserved catalytic base that is essential for phosphotransferase activity in protein kinases [26].

Previous attempts to purify the human Tyk 2 protein using multiple chromatographic steps resulted in low yields or no detectable protein. Due to the aggregation and solubility problems seen with the human isoform, orthologs were considered and an abbreviated purification protocol was implemented. This protocol entailed batch binding to GST resin for several hours, followed by a resin wash and an "on column" TEV protease cleavage step. A critical step was to introduce the ligand (Compound 1) (Figure 1) at low protein concentrations, to prevent

\section{Table 1 Representative Tyk 2 expression constructs}

\begin{tabular}{ll}
\hline Rationale & Constructs \\
\hline Orthologs & Human (hu) \\
& Mouse (mu) \\
Variation of affinity tag & His-Tev-huTyk2 (891-1185) \\
& His-Tev-huTyk2 (880-1185)-Flag \\
& GST-Thrombin-huTyk2 (870-1180) \\
& GST-Tev-muTyk2 (870-1170) \\
N- and C-terminal variations & huTyk2 (880-1185) \\
& huTyk2 (891-1185) \\
& huTyk2 (891-1175) \\
& huTyk2 (589-1185) \\
Kinase-inactive mutations & huTyk2 (870-1180) D1023A \\
& muTyk2 (870-1170) D1016A \\
\hline
\end{tabular}




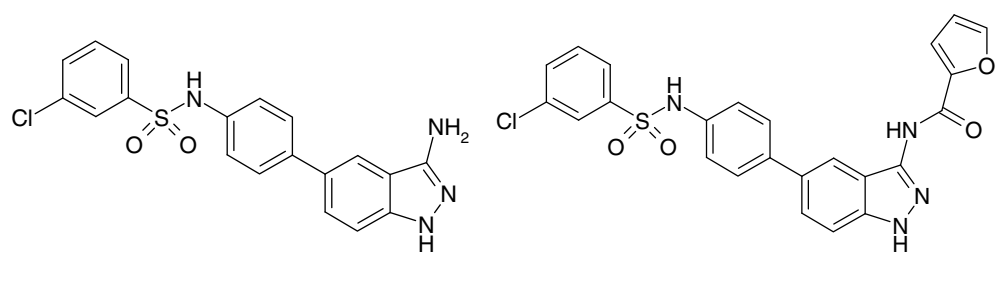

Compound 1

Compound 2

Figure 1 Structure of 3-aminoindazole inhibitors.

precipitation, and subsequently to co-concentrate the Tyk2/Compound 1 complex to a level useful for crystallization trials. Compound $1\left(\mathrm{IC}_{50} 6 \mathrm{nM}\right.$ against human Tyk2; Table 2) was one of the few inhibitors that co-crystallized with mouse Tyk2, allowing us to determine the structure of the mouse Tyk2 kinase domain. We also present the structure of Compound 2 complexed to mouse Tyk2, which was solved using inhibitor soaking methods.

\section{Proteolysis reveals stabilization of enzyme in presence of inhibitor}

Despite not directly forming crystal contacts, we found that inclusion of an ATP-competitive inhibitor was required for formation of mouse Tyk2 crystals. To understand the importance of ligand binding to the overall stability of the enzyme, we measured the Tyk2 kinase domain's susceptibility to proteolysis in the presence and absence of a ligand. Compound $2(30 \mu \mathrm{M})$ significantly increased resistance to partial proteolysis by thermolysin (Additional file 1: Figure S1). Minor processing of the kinase domain from $\sim 29 \mathrm{kDa}$ (intact) to $\sim 27 \mathrm{kDa}$ form by thermolysin is unaffected by addition of Compound 2, suggesting that its binding in the ATP site is insufficient to prevent cleavage of one of the extreme termini of our Tyk2 kinase domain construct. However, the rate of degradation of the enzyme to smaller forms $(<27 \mathrm{kDa})$ is reduced by 13 -fold (Figure 2). Like all protein kinases, the ATP binding site for Tyk2 is nestled between the $\mathrm{N}$-terminal and C-terminal lobes. Our proteolysis data suggest that the conformational flexibility of the kinase, other than a $\sim 2 \mathrm{kDa}$ portion of one terminus (likely the $\mathrm{N}$-terminus based on the disorder seen from residue 870-884 in the crystal structure described below), is decreased by the binding of these

Table 2 Inhibition of Jak enzyme activity

\begin{tabular}{lll}
\hline Kinase & Compound $\mathbf{1} \mathbf{I C}_{\mathbf{5 0}} \mathbf{( n M )}$ & Compound $\mathbf{2} \mathbf{I C}_{\mathbf{5 0}} \mathbf{( n M )}$ \\
\hline Tyk2 & $6 \pm 1$ & $<3(\mathrm{~N}=4)$ \\
Jak1 & $5 \pm 0.4$ & $10 \pm 0.1$ \\
Jak2 & $7 \pm 2$ & $4 \pm 1$ \\
Jak3 & $42 \pm 1$ & $41 \pm 3$ \\
\hline
\end{tabular}

Values are mean and standard errors.

For values below the sensitivity of the assay; the number of replicates is given. 3-aminoindazole inhibitors. The ability of Compound 1 to enable robust Tyk2 crystallization may be related, as inhibitor-induced decreased flexibility may favorably affect entropic loss during crystal nucleation and growth.

\section{Tyk2 crystal structure}

The overall structure of the mouse Tyk2 kinase domain is very similar to that of the recently reported human Tyk2 kinase domain complexed to CP-690,550 (PDB entry 3LXN; r.m.s.d $0.5 \AA$ ) (Figure 3) [27]. Two particular sequence differences between mouse and human Tyk2 may enable the crystallization of the mouse ortholog. The structure revealed that the substitution of Glu927 and Gly928 for Ala934 and Asp935 in human Tyk2 permits Gly928 to form a close, van der Waals crystal contact. Additionally, there is a potential interaction between Glu927 and Arg1132 in an adjacent molecule in the crystal lattice. Primarily due to steric clashes, a similar crystal packing would not be possible in human Tyk2. Figure 4a illustrates the sequence alignment between the mouse and human Tyk2 catalytic domains, and Figure $4 \mathrm{~b}$ provides a view of this crystal contact.

The mouse Tyk2/Compound 1 co-crystal structure is illustrated in Figure 5a. The 3-aminoindazole core serves as a canonical hinge binder, forming three hydrogen bonding interactions with hinge residues Glu972 and Val974. The inhibitor's central phenyl group linker positions the sulfonamide chlorophenyl group under the glycine rich loop. Figure $4 \mathrm{a}$ shows that the chlorophenyl moiety occupies a distinct hydrophobic pocket proximal to the DFG pocket. The placement of this moiety is guided by the sulfonamide linkage's stabilizing interactions with the $\mathrm{NH}$ backbone of Glu898 in the glycinerich loop, and conserved residues Asn1021 and Arg1020. The structure of Tyk 2 and Compound 2 is illustrated in Figure 5b. The binding mode and trajectory of the chlorophenyl is identical to that of Compound 1 and, as a result, the glycine-rich loop adopts the same conformation in both structures. The furan substituent on the hinge-binding 3-aminoindazole core was well-ordered, providing clear evidence that the inhibitor soak was successful. The furan occupies the extended hinge region, sandwiched between Arg894 and Gly977. 


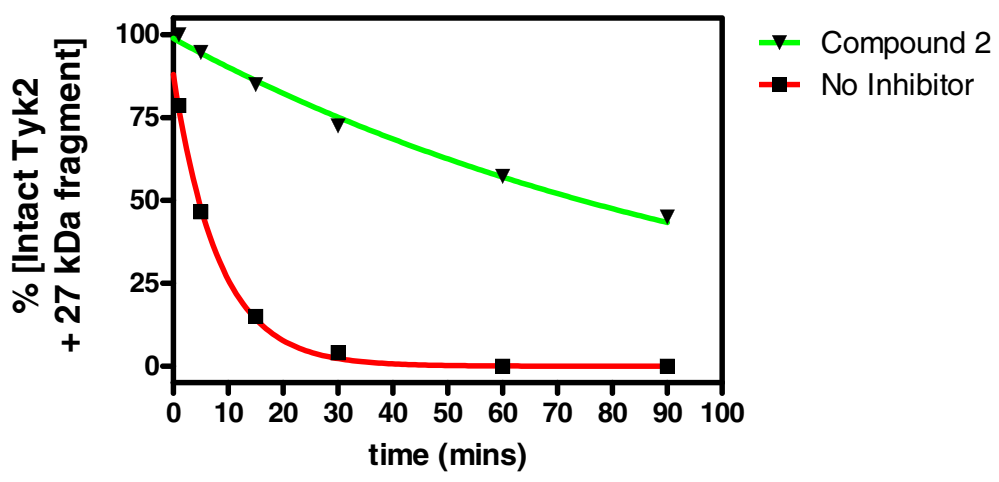

Figure 2 Tyk2 is protected from proteolysis by the addition of Compound 2. Shown are the combined, quantitated values of Tyk2 peaks of $\sim 27$ and $\sim 29 \mathrm{kDa}$ during digestion with thermolysin, in the absence or presence of $30 \mu \mathrm{M}$ Compound 2. Intact Tyk2 protein runs as $\sim 29 \mathrm{kDa}$. The $\sim 27 \mathrm{kDa}$ form produced by thermolysin digestion is unaffected by the presence of Compound 2.

One notable secondary structure difference between the co-crystallized mouse Tyk2/Compound 1 complex and the recent human Tyk2/CMP-6 complex (PDB entry 3LXP) occurs at the tip of the glycine-rich loop. An overlay shows that Compound 1 induces a $\sim 4 \AA$ upward shift in the loop (Figure 6), resulting in a more open active site conformation. In a recent review, it was suggested that the conformational dynamics of the glycine-rich loop may differ within the Jak family [28]. This may be due to sequence diversity in the glycine-rich loops of Jak1, Jak2, Jak3 and Tyk2. Specifically, in Tyk2 and Jak1, a "collapsed" glycinerich loop conformation may depend upon an interaction between a histidine residue and a proximal aspartate (His907 and Asp1023 in human Tyk2). These residues are

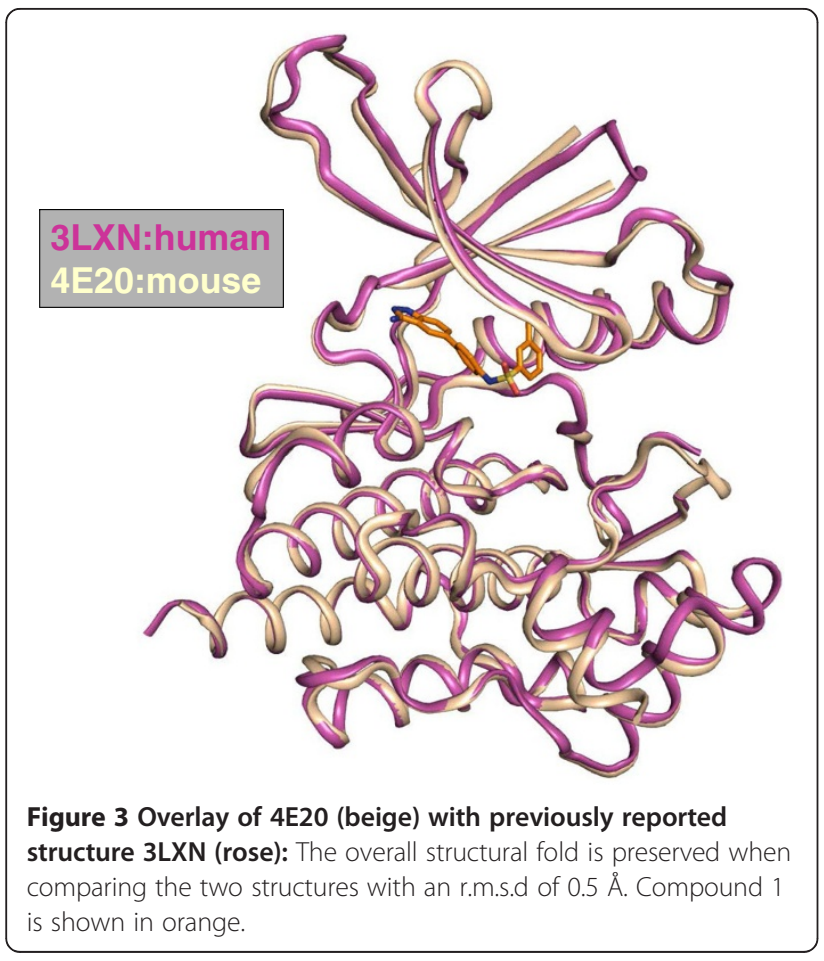

absent in Jak2 and Jak3. In the mouse Tyk2 structures, complexed to either Compound 1 or Compound 2, the steric bulk of the sulfonamide chlorophenyl moiety occupies substantial hydrophobic space under the glycine-rich loop and would potentially disrupt the His/Asp "glycinerich loop lock," thereby creating a larger active site pocket. While there are crystal contacts near the loop, we believe, based on multiple crystal structures determined with different soaked inhibitors (data not shown), that the loop conformation is driven mainly by the ligand. We cannot rule out, however, that some differences in loop conformation between human and mouse Tyk 2 may be driven by crystal packing. Despite a more open conformation, we hypothesize that mouse Tyk2 was able to crystallize with these inhibitors because the chlorophenyl moiety stabilized the flexible glycine-rich loop. Inclusion of the chloro group also improves potency by roughly 10 -fold in an enzyme activity assay (data not shown).

\section{Conclusion}

After exploring multiple expression constructs, including trials with several orthologs and mutations, we developed a method for rapid structure determination of Tyk2/inhibitor complexes suitable for iterative SBDD. We obtained crystals with a kinase-inactive form of the mouse Tyk2 catalytic domain, only in the presence of an ATP-competitive 3-aminoindazole inhibitor. This crystal form provided a robust inhibitor soaking platform that enabled structure-based drug design of Jak inhibitors. We showed by partial proteolysis that binding of a 3 -aminoindazole dramatically stabilizes Tyk2 relative to the unliganded enzyme. The resulting two crystal structures demonstrated the ability of these inhibitors to stabilize the glycine-rich loop and thus to promote conformational homogeneity. Our work indicates that compound-dependent stabilization of proteins targeted for crystallography can be a useful strategy to enable structure-based drug design. 


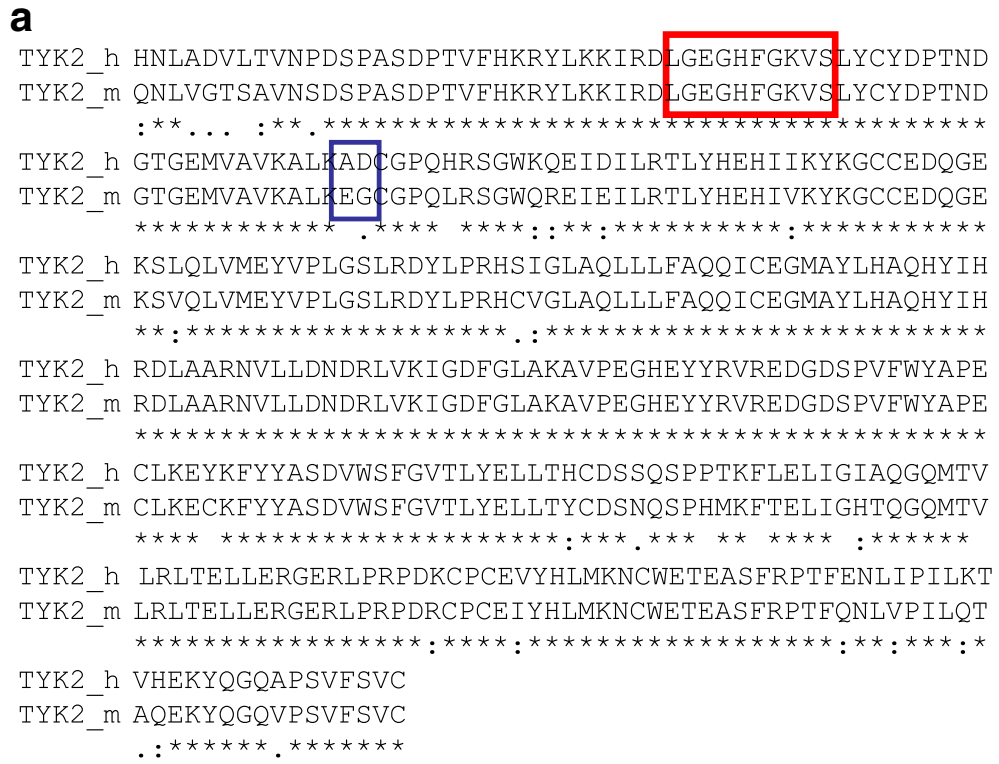

b

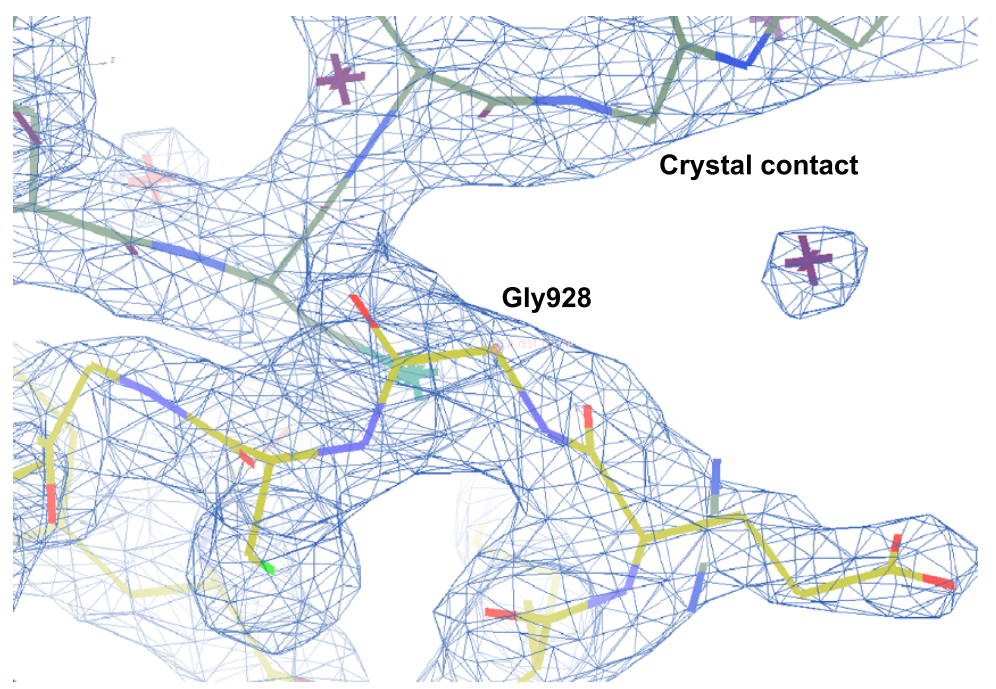

Figure 4 Tyk2 sequence variation enables novel crystal contacts. a: Sequence alignment of human and mouse Tyk2 using CLUSTALW [38]. The residues highlighted in the red box are the glycine rich loop. b: Location of mouse Tyk2 surface residue Gly928 (Asp935 in human Tyk2) permits a close, van der Waals crystal contact. (Picture generated with COOT) [36].

\section{Methods}

\section{Mouse Tyk2 cloning and purification}

Mouse Tyk2 (UNIPROT Q9R117) was cloned and expressed in $S f 9$ insect cells. The coding region of the catalytic domain of mouse (mu) Tyk2 (residues 870 1170) was PCR sub-cloned into pDONR221 using the BP reaction of the Gateway ${ }^{\circledR}$ (Invitrogen/LTI) cloning system. The muTyk2 catalytic domain was immediately preceded by a primer encoded Tobacco Etch Virus (TEV) protease cleavage site. The resulting Tev-muTyk2 (870-1170) was modified using the Quick-Change SiteDirected Mutagenesis System (Stratagene) to replace
Asp1016 with Ala. After sequence confirmation, TevmuTyk2 (870-1170) Asp1016Ala was sub-cloned into the pDEST20 expression vector (Invitrogen/LTI) using the Gateway ${ }^{\circledR}$ LR reaction to create an in-frame fusion with an amino-terminally encoded Glutathione STransferase (GST). The resulting expression plasmid, pDEST20 GST-Tev-muTyk2 (870-1170) Asp1016Ala, was confirmed by DNA sequencing. The entire expression cassette was then transferred to baculovirus. Virus production and amplifications were carried out according to Invitrogen/LTI Bac-To-Bac system instructions. 


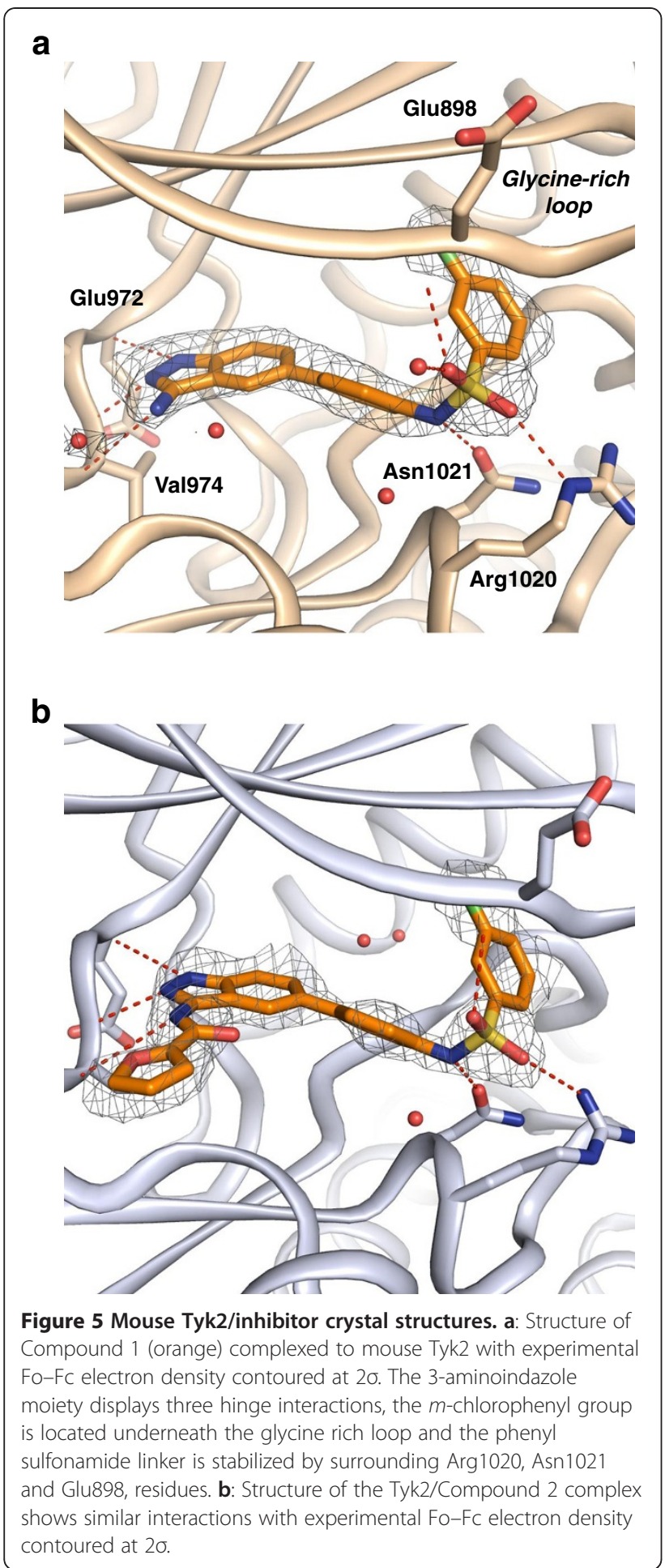

High titer virus stocks were made as recommended and used to infect $S f 9$ cells, cultured in Sf900II medium (Invitrogen/LTI) at $27.5^{\circ} \mathrm{C}$, at an estimated M.O.I of 2.5 to 5.0. Infected cells were harvested by centrifugation at $48 \mathrm{~h}$ post-infection, which was optimal for Tyk2 protein expression.
Mouse Tyk2 (870-1170) Asp1016Ala protein pellet was suspended on ice in lysis buffer containing Buffer A (50 mM HEPES pH 7.5, $500 \mathrm{mM} \mathrm{NaCl}, 10 \%$ glycerol, and $1 \mathrm{mM}$ adenosine) in addition to $2 \mathrm{X}$ protease inhibitor tablets (Roche Applied Science). The resulting mixture was sonicated three times with 20 second blasts. The mixture was then added to $10 \mathrm{~mL}$ of GST affinity resin for $2.5 \mathrm{~h}$, centrifuged at $1,000 \times \mathrm{g}$, and washed. TEV protease was added to the resin and the mixture was loaded into a column; it was incubated for $2 \mathrm{~h}$ at room temperature, and additionally overnight at $4^{\circ} \mathrm{C}$. The protein was then washed off with Buffer A and collected as monitored by $\mathrm{A}_{280}$. The pooled protein was concentrated and dialyzed overnight into $50 \mathrm{mM}$ HEPES pH 7.5, $100 \mathrm{mM} \mathrm{NaCl}, 5 \mathrm{mM}$ DTT, $1 \mathrm{mM}$ ADP. The resulting protein was pooled $(0.5 \mathrm{mg} / \mathrm{mL})$ and used directly for crystallization trials.

\section{Mouse Tyk2 crystallization}

Mouse Tyk2 protein $(0.5 \mathrm{mg} / \mathrm{mL})$ was incubated with Compound $1(0.1 \mathrm{mM})$ and concentrated to $10 \mathrm{mg} / \mathrm{mL}$. After 3-4 days, protein crystals grew using the vapor diffusion method in sitting drop plates under the following condition: 4.3-4.7 M ammonium formate, $100 \mathrm{mM}$ Tris $\mathrm{pH}$ 8.0. Crystals were subsequently used for soaking inhibitors of interest. Compound 2 was soaked into the Tyk 2 crystals by adding $1 \mu \mathrm{M}$ inhibitor (final concentration) to a $100 \mu \mathrm{L}$ well of harvest mother liquor. Crystals were frozen from mother liquor solution containing $20 \%$ glycerol.

\section{Mouse Tyk2 structure determination}

X-ray diffraction data from mouse Tyk2/Compound 1 crystals were collected at the IMCA beamline 17ID at the Advanced Photon Source in Argonne, IL. The crystals were maintained at $100 \mathrm{~K}$ with an Oxford Cryosystems Cryostream cooler during data collection. A total of 180 frames were collected at an oscillation range of $1.0^{\circ}$. The data were processed with the HKL2000 suite of programs. After determining the crystal orientation, the data were integrated with DENZO, scaled/merged with SCALEPACK, placed on an absolute scale and reduced to structure factor amplitudes with TRUNCATE. Five percent of the unique reflections were assigned randomly to the "free" set, for calculation of the free Rfactor $\left(R_{\text {free }}\right)$ [29]. The remaining $95 \%$ of the reflections constituted the "working" set for calculation of the Rfactor (R). The $\mathrm{x}$-ray diffraction data and refinement statistics are summarized in Table 2.

A maximum likelihood molecular replacement solution was determined using the program PHASER [30-32]. One Tyk2 monomer was located in the asymmetric unit, in the space group $P 3_{1} 21$. The search model was a crystal structure of Jak2 reported previously (PDB entry 


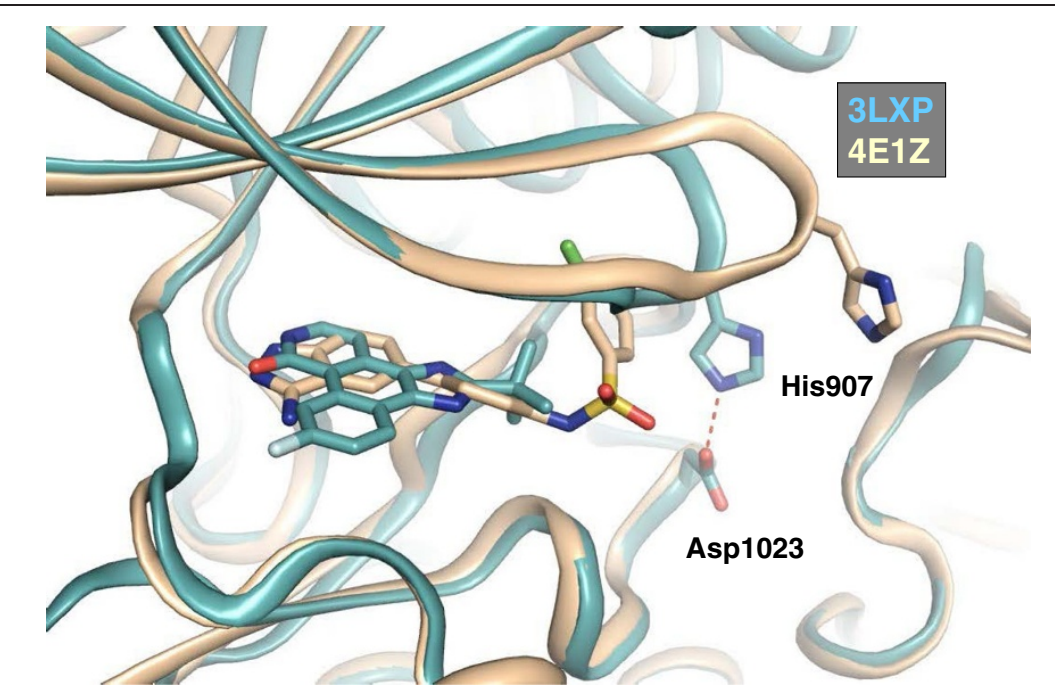

Figure 6 3-Aminoindazoles stabilize an open conformation of the Tyk2 glycine-rich loop. The chlorophenyl group of Compound 1 occupies hydrophobic pocket under the glycine rich loop. Overlay of mouse Tyk2 /Compound 2 (tan) and human Tyk2/CMP-6 (PDB entry 3LXP, cyan) structures. Deviation in the positioning of the glycine-rich loop tip is demonstrated.

2B7A). Coordinates were generated based on the molecular replacement solution. The refinement of the Tyk2/ Compound 1 complex crystal structure began with the molecular replacement solution coordinates. Rigid-body refinement was conducted by the program REFMAC [33] in the CCP4 suite of programs, which resulted in the following statistics at $2.6 \AA$ : $0.39\left(R_{\text {free }}=0.39\right)$. Experimental Tyk2 and inhibitor electron density was observed. Manual building of Compound 1 into the density was attempted using the molecular graphics program $\mathrm{O}$ [34] and examination of $2 \mathrm{Fo}-\mathrm{Fc}$ and $\mathrm{Fo}-\mathrm{Fc}$ electron-density maps. The refinement program REFMAC was used for iterative rounds of restrained refinement [33]. Final rounds of refinement were conducted using AUTOBUSTER (Global Phasing) [35], which added water molecules to the final model, resulting in the following statistics: R 0.199 $\left(\mathrm{R}_{\text {free }}=0.232\right)$. Final refinement statistics are shown in Table 3. The quality of all models was evaluated using COOT [36]. The co-crystal structure of Compound 2 complexed to Tyk2 was solved by molecular replacement using the Tyk2/Compound 1 structure as a probe. An ori-

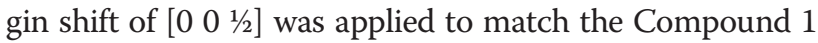
coordinates. DETWIN [37] was used with a twinning fraction of 0.24 to improve refinement statistics. Final rounds of refinement were conducted using AUTOBUSTER (Global Phasing) [35]. Final refinement statistics are listed in Table 3.

\section{Time-resolved fluorescence resonance energy transfer (trFRET) kinase activity assays \\ Tyk2}

$6 \mathrm{nM}$ purified human Tyk2 enzyme (residues 880-1185; expressed in $S f 9$ cells with a cleavable $N$-terminal
Table 3 Crystallographic statistics for Tyk2/Compound 1 and Tyk2/Compound 2 complexes

\begin{tabular}{|c|c|c|}
\hline Structure & $\begin{array}{l}\text { Tyk2/ } \\
\text { Compound } 1\end{array}$ & $\begin{array}{l}\text { Tyk2/ } \\
\text { Compound } 2\end{array}$ \\
\hline PDB entry & $4 \mathrm{E} 20$ & $4 \mathrm{E} 1 \mathrm{Z}$ \\
\hline \multicolumn{3}{|l|}{ Data Collection } \\
\hline Resolution $(\AA)$ & $50.0-2.60$ & $20.0-2.50$ \\
\hline (Highest shell, $\AA$ ) & $2.64-2.60$ & $2.54-2.50$ \\
\hline Space Group & $P 3,21$ & $P 3_{1} 21$ \\
\hline Unit Cell Lengths $(a, b, c ; \AA)$ & $67.3,67.3,154.9$ & $68.0,68.0,153.0$ \\
\hline Unique reflections & 13,121 & 14,608 \\
\hline Mosaicity $\left({ }^{\circ}\right)$ & 0.247 & 0.642 \\
\hline \multicolumn{3}{|l|}{ Overall Statistics (Highest Shell) } \\
\hline $\mathrm{R}_{\text {sym }}(\%)$ & $0.085(0.511)$ & $0.055(0.480)$ \\
\hline $1 / \sigma_{l}$ & $8.9(2.5)$ & $9.2(2.3)$ \\
\hline Data completeness (\%) & $99.9(100)$ & $98.3(97.4)$ \\
\hline Mean multiplicity & $6.9(5.1)$ & $6.8(4.7)$ \\
\hline \multicolumn{3}{|l|}{ Refinement } \\
\hline Reflections used in refinement & 13,072 & 13,973 \\
\hline $\mathrm{R}_{\text {cryst }}(\%)$ & 19.9 & 19.8 \\
\hline$R_{\text {free }}(\%)$ & 23.2 & 24.3 \\
\hline $\begin{array}{l}\text { R.m.s. deviations, bond lengths } \\
(\AA) \text {, bond angles }\left(^{\circ}\right)\end{array}$ & $0.010,1.15$ & $0.010,1.20$ \\
\hline \multicolumn{3}{|l|}{ Ramachadran plot } \\
\hline Most favored (\%) & 96.4 & 96.1 \\
\hline Allowed (\%) & 2.2 & 3.2 \\
\hline Disallowed (\%) & 1.5 & 0.7 \\
\hline
\end{tabular}




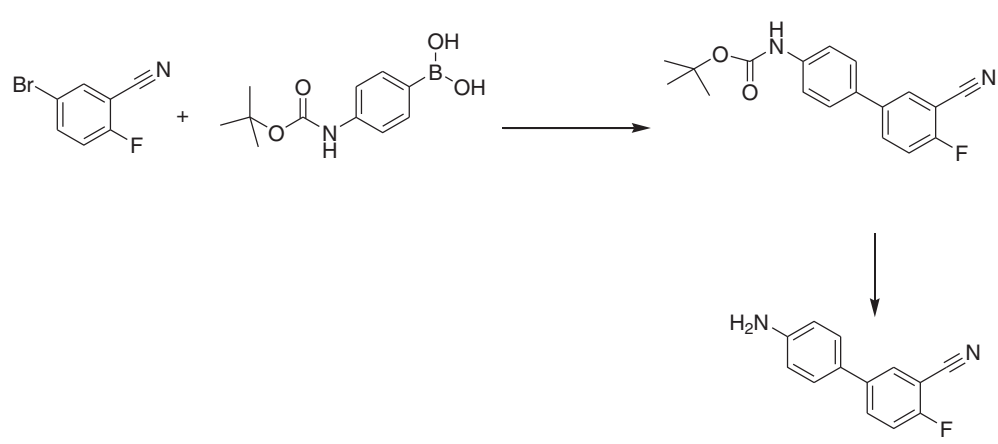

Figure 7 Step a. Synthetic route to 4-(3-Cyano-4-fluoro-phenyl) aniline.

histidine tag and a $C$-terminal Flag tag and purified by affinity chromatography) was mixed with $2 \mu \mathrm{M}$ peptide substrate (biotin-TYR1, sequence: Biotin-(Ahx)-GAEEEIYAAFFA$\mathrm{COOH})$ at varying concentrations of inhibitor in reaction buffer: $50 \mathrm{mM}$ MOPSO $\mathrm{pH}$ 6.5, $10 \mathrm{mM} \mathrm{MgCl}_{2}$, $2 \mathrm{mM} \mathrm{MnCl}_{2}, 1 \mu \mathrm{M}$ ATP, $2.5 \mathrm{mM}$ DTT, 0.01\% BSA, and $0.1 \mathrm{mM} \mathrm{Na} \mathrm{VO}_{4}$. After $60 \mathrm{~min}$ incubation at room temperature, the reaction was quenched by addition of EDTA (final conc. $100 \mu \mathrm{M}$ ) and developed by addition of revelation reagents (final approximate concentrations: $30 \mathrm{mM}$ HEPES $\mathrm{pH} 7.0,0.06 \%$ BSA, 0.006\% Tween-20, 0.24 M KF, 80 ng/mL PT66K (europium-labelled anti-phosphotyrosine antibody, cat \#61T66KLB, Cisbio, Bedford, MA) and $3.12 \mu \mathrm{g} / \mathrm{mL}$ SAXL (Phycolink streptavidin-allophycocyanin acceptor, cat \#PJ25S, Prozyme, San Leandro, CA)). The developed reaction was incubated in the dark either at $4^{\circ} \mathrm{C}$ overnight or at room temperature for $\sim 1 \mathrm{~h}$, then read with a time-resolved fluorescence detector (Rubystar, BMG Labtech) using a $337 \mathrm{~nm}$ laser for excitation and emission wavelengths of $620 \mathrm{~nm}$ and $665 \mathrm{~nm}$. Within the linear range of the assay, this signal is directly related to phosphorylated product and was used to calculate $\mathrm{IC}_{50}$ values. Typically, seven-point inhibitor dilutions (5-fold; from $50 \mu \mathrm{M}$ to $0.0032 \mu \mathrm{M}$ ) were used. $\mathrm{IC}_{50}$ values were calculated by fitting the following equation:

$$
Y=Y_{\max } * I C_{50} /\left(I C_{50}+[I]\right)
$$

where [I] is total inhibitor concentration, $\mathrm{Y}$ is the percentage of activity (relative to that seen in no-inhibitor control) at a given inhibitor concentration, and $\mathrm{Y}_{\max }$ is the maximum activity generated in the absence of inhibitor.

\section{Jak1, Jak2 and Jak3}

Purified Jak2 and Jak3 were purchased from Upstate/ Millipore (cat numbers \#14-640 and \#14-629). Jak1 (residues 845-1142) was expressed in Sf9 cells as a GST fusion protein and purified in-house, and used in trFRET kinase assays in the reaction buffer described above. Jak1 and Jak3 assays used substrate peptide biotin-TYR2 (Biotin-(Ahx)-AEEEYFFLFA-amide), while the Jak2 assay used biotin-TYR1 (Biotin-(Ahx)-GAEEEIYAAFFA-COOH).

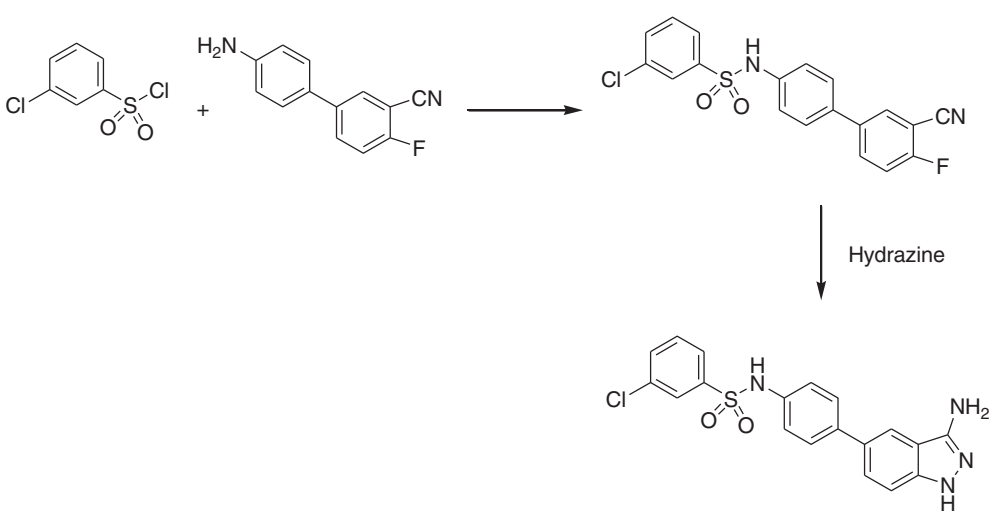

Figure 8 Step b. Synthetic route to Compound 1. 


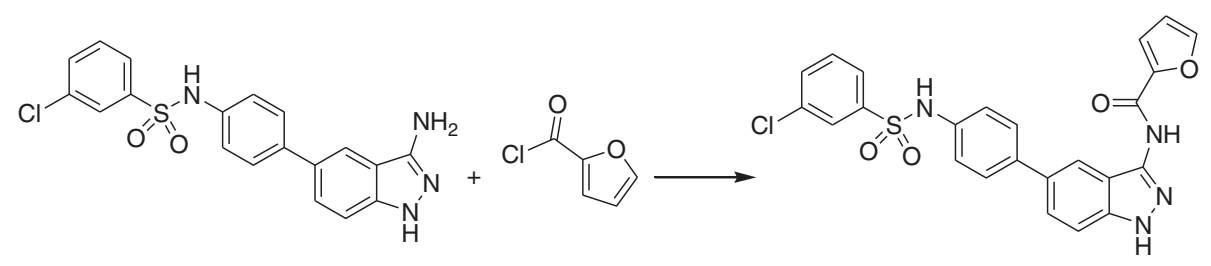

Figure 9 Synthetic route to Compound 2.

\section{Proteolysis experiments}

Mouse Tyk2 kinase domain $(0.25 \mathrm{mg} / \mathrm{mL}$, residues 870 1170; Asp1016Ala mutant) was incubated for 0 to $90 \mathrm{~min}$ utes with thermolysin $(0.25 \mathrm{mg} / \mathrm{mL})$ at room temperature in $50 \mathrm{mM}$ HEPES pH 6.7, $150 \mathrm{mM} \mathrm{NaCl}, 5 \%$ glycerol, and $2.5 \mathrm{mM} \mathrm{CaCl}_{2}$ in the presence and absence of $30 \mu \mathrm{M}$ Compound 2. EDTA (final conc $100 \mathrm{mM}$ ) was used as stop solution to quench the proteolysis reactions. Samples were separated by use of a Caliper LC90 system and the remaining substrate and product bands were quantitated. Intact (undigested) Tyk2 ran in this system at $\sim 29 \mathrm{kDa}$. Addition of thermolysin yielded a partial digestion product of $\sim 27 \mathrm{kDa}$ (Additional file 1: Figure 1) within 5 minutes, which was unaffected by addition of Compound 2. Subsequent degradation products were strongly influenced by the presence of Compound 2 (Additional file 1: Figure S1). Therefore the sum of the intensities of the 29 and $27 \mathrm{kDa}$ peaks was used as a measure of inhibitor-dependent resistance to thermolysin digestion. Addition of Compound 2 did not alter the measurable digestion of a BSA control by thermolysin in the same buffer (Additional file 1: Figure S2a), indicating that Compound 2 was not an inhibitor of thermolysin protease activity. Thermolysin amounts were found to be unchanged over the course of the experiment, providing a convenient loading control (Additional file 1: Figure S2b).

\section{Compound synthesis}

$\mathrm{N}$-[4-(3-Amino-1H-indazol-5-yl)-phenyl]-3-chlorobenzenesulfonamide (Compound 1)

(See Figures 1, 8)

Step a. 4-(3-Cyano-4-fluoro-phenyl) aniline (Figure 7)

A mixture of 5-bromo-2-fluorobenzonitrile (2.185 g, $10.93 \mathrm{mmol}), 4$-(tert-butoxycarbonylamino) phenylboronic acid (2.59 g, $10.93 \mathrm{mmol})$, tetrakis(triphenylphosphine)palladium $(0.758 \mathrm{~g}, 0.656 \mathrm{mmol})$ and cesium carbonate (10.68 g, $32.8 \mathrm{mmol})$ in 1,2dimethoxyethane $(40.0 \mathrm{ml})$ and water $(20.00 \mathrm{ml})$ was heated at $80^{\circ} \mathrm{C}$, under $\mathrm{N}_{2}$ for about 2.5 hours. to give a dark red solution. The reaction mixture was diluted with ethyl acetate $(100 \mathrm{~mL})$ and water $(100 \mathrm{~mL})$ then stirred for about 10 minutes. The aqueous layer was separated and re-extracted with ethyl acetate
$(2 \times 50 \mathrm{~mL})$. The combined organic phases were washed with water $(3 \times 50 \mathrm{~mL})$ then dried over anhydrous magnesium sulfate and filtered. The solvent was removed to yield a brown solid (4.12 g) which was triturated with boiling $30-60^{\circ} \mathrm{C}$ petroleum ether $(40 \mathrm{~mL})$, cooled and collected. This solid was further washed with $30-60^{\circ} \mathrm{C}$ petroleum ether $(2 \times 20 \mathrm{~mL})$ and dried to yield crude $N$-tert-butoxycarbonyl-4-(3-cyano4-fluoro-phenyl) aniline as a pale brown solid (3.86 g). The crude protected aniline was dissolved in dichloromethane $(80 \mathrm{~mL})$ then treated with trifluoroacetic acid (14.0 mL, $182 \mathrm{mmol}$ ) and stirred at ambient temperature for about 3 hours. Water $(100 \mathrm{~mL})$ was added and the aqueous acidic layer was separated. The remaining organic layer was further extracted with aqueous hydrochloric acid ( $5 \mathrm{~N}$, $4 \times 60 \mathrm{~mL}$ ). The combined acidic aqueous layers were washed with dichloromethane $(3 \times 50 \mathrm{~mL})$, cooled with ice then basified by the addition of solid sodium hydroxide while maintaining a temperature of below $15^{\circ} \mathrm{C}$. The resulting aqueous layer was extracted with ethyl acetate $(4 \times 75 \mathrm{~mL})$ and the combined organic layers were washed with water $(3 \times 80 \mathrm{~mL})$, dried over anhydrous magnesium sulphate, filtered and concentrated to yield a mauve solid $(2.1 \mathrm{~g})$. This was crystallized from ethyl acetate $(8 \mathrm{~mL})$ and $30-60^{\circ} \mathrm{C}$ petroleum ether $(32 \mathrm{~mL})$, filtered, washed with $30-60^{\circ}$ $\mathrm{C}$ petroleum ether $(2 \times 15 \mathrm{~mL})$ and dried to yield 4 - $(3$ cyano-4-fluoro-phenyl) aniline as a pale mauve powdery solid (1.84 g, 80\% yield); ${ }^{1} \mathrm{H}$ NMR (DMSO- $\left.d_{6}\right) \delta 6.62$ (dd, $2 \mathrm{H}), 7.41$ (dd, $2 \mathrm{H}), 7.50(\mathrm{t}, 1 \mathrm{H}), 7.93(\mathrm{~m}, 1 \mathrm{H})$, 8.06 (dd, $1 \mathrm{H}$ ); LC/MS (5-60\% gradient of acetonitrile in $10 \mathrm{mM}$ aqueous ammonium acetate over $1.5 \mathrm{~min}$, 60-95\% gradient of acetonitrile in $10 \mathrm{mM}$ aqueous ammonium acetate over $2.5 \mathrm{~min}$ with a hold at $95 \%$ acetonitrile for $1.2 \mathrm{~min}(1.3 \mathrm{~mL} / \mathrm{min}$ flow rate) using a Vydac Genesis C8 column $(4.6 \times 30 \mathrm{~mm}, 4 \mu \mathrm{m}$ particle $)$ with diode array (DAD), evaporative light scattering (ELSD) and positive/negative electrospray ionization detection) $\mathrm{R}_{\mathrm{t}}=2.43 \mathrm{~min}$; $\mathrm{MS} \mathrm{m} / z: 254\left(\mathrm{M}+\mathrm{H}+\mathrm{CH}_{3} \mathrm{CN}\right)^{+}$. Step b. $N$-[4-(3-Amino- $1 H$-indazol-5-yl)-phenyl]-3chloro-benzenesulfonamide (Compound 1) (See Figure 8)

3-Chlorobenzenesulfonyl chloride $(0.563 \mathrm{~mL}$, $4.0 \mathrm{mmol}, 2$ equivalents, Aldrich) was added to a 
mixture of 4-(3-cyano-4-fluoro-phenyl)aniline (424 mg, $2.0 \mathrm{mmol})$ in 1,4-dioxane $(10 \mathrm{~mL})$ and $N, N$ -

diisopropylethylamine $(1.04 \mathrm{~mL})$. The resulting mixture was stirred at ambient temperature for about 16 hours then dispensed into a microwaveable tube and anhydrous hydrazine $(2 \mathrm{~mL})$ was added. The tube was sealed and heated at $140^{\circ} \mathrm{C}$ for 15 minutes in a microwave. The solvent was removed under reduced pressure and the residue was purified by reverse-phase preparative chromatography (using a gradient of 5 to $100 \%$ acetonitrile in $0.05 \mathrm{~N}$ aqueous ammonium acetate) followed by chromatography over silica gel using a mixture of 9:1 dichloromethane:methanol as the eluent to afford $N$-[4-(3-amino-1 H-indazol-5-yl)phenyl]-3-chloro-benzenesulfonamide (113 mg, 14\% yield); ${ }^{1} \mathrm{H}$ NMR (DMSO- $\left.d_{6}\right) \delta 5.39(\mathrm{~m}, 2 \mathrm{H}), 7.17(\mathrm{~m}$, $2 \mathrm{H}), 7.26(\mathrm{~d}, 1 \mathrm{H}), 7.47(\mathrm{dd}, 1 \mathrm{H}), 7.56(\mathrm{~m}, 3 \mathrm{H}), 7.72$ (m, $2 \mathrm{H}), 7.79$ (t, $1 \mathrm{H}), 10.41(\mathrm{~s}, 1 \mathrm{H}), 11.40(\mathrm{~m}, 1 \mathrm{H})$; LC/MS (5-95\% gradient of acetonitrile in $10 \mathrm{mM}$ aqueous ammonium acetate over 2.9 min with a hold at $95 \%$ acetonitrile for $18 \mathrm{~min}(1.3 \mathrm{~mL} / \mathrm{min}$ flow rate) using a Zorbax XDB C18 column $(4.6 \times 50 \mathrm{~mm}, 5 \mu \mathrm{m}$ particle) with diode array (DAD), evaporative light scattering (ELSD) and positive/negative electrospray ionization detection) $\mathrm{R}_{\mathrm{t}}=2.32 \mathrm{~min}$; MS $\mathrm{m} / z: 399,401$ $(\mathrm{M}+\mathrm{H})^{+}$.

\section{Furan-2-carboxylic acid \{5-[4-(3-chloro- benzenesulfonylamino)-phenyl]-1 H-indazol-3-yl\}amide (Compound 2)}

(See Figure 9)

2-Furoyl chloride $(0.123 \mathrm{~mL}, 1.254 \mathrm{mmol}$, Aldrich) was added dropwise to a solution of $\mathrm{N}$-[4-(3-amino- $1 \mathrm{H}$ indazol-5-yl)-phenyl]-3-chloro-benzenesulfonamide (250 mg, $0.627 \mathrm{mmol}$, Compound 1) in pyridine $(6.5 \mathrm{~mL})$ at about $0^{\circ} \mathrm{C}$. The reaction was allowed to warm to ambient temperature and stirred for 18 hours. Ethyl acetate $(20 \mathrm{~mL})$ and methanol $(5 \mathrm{~mL})$ were added to the reaction mixture and the resulting solution was washed with water $(3 \times 10 \mathrm{~mL})$. The organic layer was dried over anhydrous magnesium sulphate and concentrated under reduced pressure. The residue was purified over silica gel eluting with a gradient of $0-100 \%$ ethyl acetate in heptane to afford furan-2-carboxylic acid \{5[4-(3-chloro-benzenesulfonylamino)-phenyl]-1 H-indazol3-ylsamide (195 mg, 63\% yield); ${ }^{1} \mathrm{H}$ NMR (DMSO- $\left.d_{6}\right) \delta$ $6.71(\mathrm{~s}, 1 \mathrm{H}), 7.18(\mathrm{~d}, 2 \mathrm{H}), 7.46(\mathrm{~d}, 1 \mathrm{H}), 7.54(\mathrm{~m}, 5 \mathrm{H})$, $7.64(\mathrm{~m}, 2 \mathrm{H}), 7.73(\mathrm{~s}, 1 \mathrm{H}), 7.84(\mathrm{~s}, 1 \mathrm{H}), 7.91(\mathrm{~s}, 1 \mathrm{H})$, $10.42(\mathrm{~s}, 1 \mathrm{H}), 10.71(\mathrm{~s}, 1 \mathrm{H}), 12.79(\mathrm{~s}, 1 \mathrm{H}) ;$ LC/MS (5-95\% gradient of acetonitrile in $10 \mathrm{mM}$ aqueous ammonium acetate over $2.9 \mathrm{~min}$ with a hold at $95 \%$ acetonitrile for $18 \mathrm{~min}(1.3 \mathrm{~mL} / \mathrm{min}$ flow rate) using a Zorbax XDB C18 column $(4.6 \times 50 \mathrm{~mm}, 5 \mu \mathrm{m}$ particle $)$ with diode array (DAD), evaporative light scattering (ELSD) and positive/negative electrospray ionization detection) $\mathrm{R}_{\mathrm{t}}=2.47 \mathrm{~min} ; \mathrm{MS} \mathrm{m} / \mathrm{z}: 491,493\left(\mathrm{M}-\mathrm{H}^{+}\right)^{-}$.

\section{Additional file}

Additional file 1: Figure S1. Caliper LC90 "virtual gel" depiction of chromatography results with Tyk2 proteolysis using thermolysin. $0.25 \mathrm{mg} / \mathrm{mL}$ Tyk2 kinase domain was incubated with thermolysin at room temperature in $50 \mathrm{mM}$ Hepes pH 6.7, 150mM NaCl, 5\% Glycerol, $2.5 \mathrm{mM} \mathrm{CaCl} 2$ in the presence and absence of Compound 2. EDTA (final conc $100 \mathrm{mM}$ ) was used as stop solution to quench the proteolysis reactions.

$8 \mu \mathrm{L}$ of this reaction were subsequently run in the Caliper LC90 "gel chip". Small processing of Tyk2 from $\sim 29 \mathrm{kDa}$ (intact) to $\sim 27 \mathrm{kDa}$ form by thermolysin is unaffected by addition of Compound 2, suggesting that its binding in the ATP site is insufficient to prevent processing of one of the extreme termini of our Tyk2 construct. In the absence of inhibitor,

a $20 \mathrm{kDa}$ fragment is generated after $\sim 1-5$ minutes and subsequently degraded. This fragment is undetectable in the similar digestion in the presence of Compound 2. Quantitated values of Tyk2 peaks of $\sim 27$ and $\sim 29$ $\mathrm{kDa}$ during digestion with thermolysin, in the absence or presence of $30 \mu \mathrm{M}$ Compound 2 were used to monitor overall degradation rates in Figure 2. Figure S2: (a) Addition of Compound 2 did not alter the measurable digestion of a BSA control (at $1 \mathrm{mg} / \mathrm{mL}$ in reaction) by thermolysin $(0.5 \mathrm{mg}$ / $\mathrm{mL}$ in reaction) in $50 \mathrm{mM}$ Hepes $\mathrm{pH} 6.7,150 \mathrm{mM} \mathrm{NaCl}, 5 \%$ Glycerol, $2.5 \mathrm{mM}$ $\mathrm{CaCl}$. This indicates that Compound 2 was not an inhibitor of thermolysin protease activity. Quantitated values from $2 \mu \mathrm{L}$ injected onto the Caliper LC90 are shown scaled relative to starting concentration. (b) Thermolysin levels were found to be essentially unchanged over the course of the experiment shown in Additional file 1: Figure S1 and were not affected by the addition of Compound 2. Table S1: Crystallographic constructs attempted with expression and solubility assessments.

\section{Competing interests}

The authors declare that they have no competing interests.

\section{Authors' contributions}

MAA and DWB led the Tyk2 structural biology sub-team and participated in construct design. RWD contributed to construct design. MAA and DWB solved and refined the reported crystal structures. SS made the majority of the constructs and performed some of the protein expression. DB, DM and VP purified protein, set up crystallizations, and collected diffraction data. MT and GO contributed protein characterization and purification for enzymatic assays. RS provided construct design and protein expression oversight and participated in construct design. RVT participated in construct design and supervised the structural biology and enzymology teams. ERG supervised the enzyme screening and performed the proteolysis experiments. JV, KW and NW jointly led the Tyk2 project team. NM, LW and AB conceived and synthesized the compounds. MAA and ERG jointly prepared the manuscript, in consultation with all of the co-authors. All authors have read and approved this manuscript.

\section{Acknowledgements}

The authors thank Ayome Abibi, Richard Petrillo, Andrew Gagnon, Carl Hollins, Silvia Kwak, Ahmad Saadat, Christopher Harris, and John Mankovich for their technical assistance and helpful discussions. We also thank Vincent Stoll and Kenton Longenecker for helpful discussions regarding construct design and assistance in data collection at APS. Use of the IMCA-CAT beamline 17-ID at the Advanced Photon Source was supported by the companies of the Industrial Macromolecular Crystallography Association through a contract with Hauptman-Woodward Medical Research Institute. Use of the Advanced Photon Source was supported by the U.S. Department of Energy, Office of Science, Office of Basic Energy Sciences, under Contract No. DE-ACO2-06CH11357.

\section{Author details}

'Department of Molecular \& Cellular Pharmacology, Abbott Laboratories, Worcester, MA, USA. ${ }^{2}$ Present Address: D. E. Shaw Research, New York, NY, USA. ${ }^{3}$ Department of Chemistry, Abbott Laboratories, Worcester, MA, USA. 
${ }^{4}$ Present Address: Vertex Pharmaceuticals, Cambridge, MA, USA. ${ }^{5}$ Present Address: Department of Physical Biochemistry, Biogen Idec, Cambridge, MA, USA. ${ }^{6}$ Department of Biologics, Abbott Laboratories, Worcester, MA, USA. ${ }^{7}$ Department of Molecular Cell Biology, Harvard University, Cambridge, MA, USA. ${ }^{8}$ Advanced Technologies, Abbott Laboratories, Abbott Park, IL, USA.

Received: 12 March 2012 Accepted: 27 July 2012

Published: 20 September 2012

\section{References}

1. Darnell JE Jr: STATs and gene regulation. Science 1997, 277(5332): 1630-1635.

2. Horvath CM: STAT proteins and transcriptional responses to extracellular signals. Trends Biochem Sci 2000, 25(10):496-502.

3. Yamaoka K, Saharinen P, Pesu M, Holt VE 3rd, Silvennoinen O, O'Shea JJ: The Janus kinases (Jaks). Genome Biol 2004, 5(12):253.

4. Watford WT, Hissong BD, Bream JH, Kanno Y, Muul L, O'Shea JJ: Signaling by IL-12 and IL-23 and the immunoregulatory roles of STAT4. Immunol Rev 2004, 202:139-156.

5. Minegishi Y, Saito M, Morio T, Watanabe K, Agematsu K, Tsuchiya S, Takada H, Hara T, Kawamura N, Ariga T, et al: Human tyrosine kinase 2 deficiency reveals its requisite roles in multiple cytokine signals involved in innate and acquired immunity. Immunity 2006, 25(5):745-755.

6. Ortmann RA, Cheng T, Visconti R, Frucht DM, O'Shea Jj: Janus kinases and signal transducers and activators of transcription: their roles in cytokine signaling, development and immunoregulation. Arthritis Res 2000, 2(1):16-32.

7. Karaghiosoff M, Neubauer H, Lassnig C, Kovarik P, Schindler H, Pircher $H$, McCoy B, Bogdan C, Decker T, Brem G, et al: Partial impairment of cytokine responses in Tyk2-deficient mice. Immunity 2000, 13(4):549-560.

8. Liu KD, Gaffen SL, Goldsmith MA, Greene WC: Janus kinases in interleukin2-mediated signaling: JAK1 and JAK3 are differentially regulated by tyrosine phosphorylation. Curr Biol 1997, 7(11):817-826.

9. Nosaka T, van Deursen JM, Tripp RA, Thierfelder WE, Witthuhn BA, McMickle AP, Doherty PC, Grosveld GC, Ihle JN: Defective lymphoid development in mice lacking Jak3. Science 1995, 270(5237):800-802.

10. Langrish CL, McKenzie BS, Wilson NJ, de Waal Malefyt R, Kastelein RA, Cua DJ: IL-12 and IL-23: master regulators of innate and adaptive immunity. Immunol Rev 2004, 202:96-105.

11. Murray PJ: The JAK-STAT signaling pathway: input and output integration. J Immunol 2007, 178(5):2623-2629.

12. Kudlacz E, Perry B, Sawyer P, Conklyn M, McCurdy S, Brissette W, Flanagan And M, Changelian P: he novel JAK-3 inhibitor CP-690550 is a potent immunosuppressive agent in various murine models. Am J Transplant 2004, 4(1):51-57.

13. Changelian PS, Flanagan ME, Ball DJ, Kent CR, Magnuson KS, Martin WH, Rizzuti BJ, Sawyer PS, Perry BD, Brissette WH, et al: Prevention of organ allograft rejection by a specific Janus kinase 3 inhibitor. Science 2003, 302(5646):875-878.

14. Ghoreschi K, Laurence A, O'Shea JJ: Selectivity and therapeutic inhibition of kinases: to be or not to be? Nat Immunol 2009, 10(4):356-360

15. West K: CP-690550, a JAK3 inhibitor as an immunosuppressant for the treatment of rheumatoid arthritis, transplant rejection, psoriasis and other immune-mediated disorders. Curr Opin Investig Drugs 2009, 10 (5):491-504.

16. Sorbera LA, Serradell N, Bolós J, Rosa E, Bozzo J: CP-690550. Drugs of the Future 2007, 32(8):674-680.

17. Tanaka Y, Suzuki M, Nakamura H, Toyoizumi S, Zwillich SH: Phase II study of tofacitinib $(\mathrm{CP}-690,550)$ combined with methotrexate in patients with rheumatoid arthritis and an inadequate response to methotrexate. Arthritis Care Res (Hoboken) 2011, 63(8):1150-1158.

18. Kremer JM, Cohen S, Wilkinson BE, Connell CA, French $J$, Gomez-Reino J, Gruben D, Kanik KS, Krishnaswami S, Pascual-Ramos V, et al: A Phase 2B dose-ranging study of the oral JAK inhibitor tofacitinib (CP-690,550) versus placebo in combination with background methotrexate in patients with active rheumatoid arthritis and inadequate response to methotrexate alone. Arthritis Rheum 2012

19. Fleischmann R, Cutolo M, Genovese MC, Lee EB, Kanik KS, Sadis S, Connell CA, Gruben D, Krishnaswami S, Wallenstein $G$, et al: Phase 2B dose-ranging study of the oral JAK inhibitor tofacitinib (CP-690,550) or adalimumab monotherapy versus placebo in patients with active rheumatoid arthritis with an inadequate response to DMARDs. Arthritis Rheum 2012.
20. Verstovsek S, Kantarjian H, Mesa RA, Pardanani AD, Cortes-Franco J, Thomas DA, Estrov Z, Fridman JS, Bradley EC, Erickson-Viitanen S, et al: Safety and efficacy of INCB018424, a JAK1 and JAK2 inhibitor, in myelofibrosis. N Engl J Med 2010, 363(12):1117-1127.

21. Lankford CS, Frucht DM: A unique role for IL-23 in promoting cellular immunity. J Leukoc Biol 2003, 73(1):49-56.

22. Oyamada A, Ikebe H, Itsumi M, Saiwai H, Okada S, Shimoda K, I wakura Y, Nakayama Kl, Iwamoto Y, Yoshikai Y, et al: Tyrosine kinase 2 plays critical roles in the pathogenic CD4 T cell responses for the development of experimental autoimmune encephalomyelitis. J Immunol 2009, 183(11):7539-7546.

23. Spach KM, Noubade R, McElvany B, Hickey WF, Blankenhorn EP, Teuscher C: A single nucleotide polymorphism in Tyk2 controls susceptibility to experimental allergic encephalomyelitis. J Immunol 2009, 182(12):7776-7783.

24. Boggon TJ, Li Y, Manley PW, Eck MJ: Crystal structure of the Jak3 kinase domain in complex with a staurosporine analog. Blood 2005, 106(3):996-1002.

25. Lucet IS, Fantino E, Styles M, Bamert R, Patel O, Broughton SE, Walter M, Burns $C$, Treutlein $H$, Wilks AF, et al: The structural basis of Janus kinase 2 inhibition by a potent and specific pan-Janus kinase inhibitor. Blood 2006, 107(1):176-183.

26. Madhusudan, Trafny EA, Xuong NH, Adams JA, Ten Eyck LF, Taylor SS, Sowadski JM: CAMP-dependent protein kinase: crystallographic insights into substrate recognition and phosphotransfer. Protein Sci 1994, 3(2):176-187.

27. Chrencik JE, Patny A, Leung IK, Korniski B, Emmons TL, Hall T, Weinberg RA, Gormley JA, Williams JM, Day JE, et al: Structural and Thermodynamic Characterization of the TYK2 and JAK3 Kinase Domains in Complex with CP-690550 and CMP-6. J Mol Biol 2010. 400(3):413-433.

28. Alicea-Velazquez NL, Boggon TJ: The use of structural biology in Janus kinase targeted drug discovery. Curr Drug Targets 2011, 12(4):546-555.

29. Brunger AT: Free $R$ value: a novel statistical quantity for assessing the accuracy of crystal structures. Nature 1992, 355(6359):472-475.

30. McCoy AJ, Grosse-Kunstleve RW, Storoni LC, Read RJ: Likelihood-enhanced fast translation functions. Acta Crystallogr D Biol Crystallogr 2005, 61(Pt 4):458-464.

31. Read RJ: Pushing the boundaries of molecular replacement with maximum likelihood. Acta Crystallogr D Biol Crystallogr 2001, 57(Pt 10):1373-1382.

32. Storoni LC, MCCoy AJ, Read RJ: Likelihood-enhanced fast rotation functions. Acta Crystallogr D Biol Crystallogr 2004, 60(Pt 3):432-438.

33. Murshudov GN, Vagin AA, Dodson EJ: Refinement of macromolecular structures by the maximum-likelihood method. Acta Crystallogr D Biol Crystallogr 1997, 53(Pt 3):240-255.

34. Jones TA, Zou JY, Cowan SW, Kjeldgaard M: Improved methods for building protein models in electron density maps and the location of errors in these models. Acta Crystallogr A 1991, 47(Pt 2):110-119.

35. Blanc E, Roversi P, Vonrhein C, Flensburg C, Lea SM, Bricogne G: Refinement of severely incomplete structures with maximum likelihood in BUSTERTNT. Acta Crystallogr D Biol Crystallogr 2004, 60(Pt 12 Pt 1):2210-2221.

36. Emsley P, Cowtan K: Coot: model-building tools for molecular graphics. Acta Crystallogr D Biol Crystallogr 2004, 60(Pt 12 Pt 1):2126-2132

37. Collaborative Computational Project N: The CCP4 suite: programs for protein crystallography. Acta Crystallogr D Biol Crystallogr 1994, 50(Pt 5):760-763.

38. Larkin MA, Blackshields G, Brown NP, Chenna R, McGettigan PA, McWilliam $H$, Valentin F, Wallace IM, Wilm A, Lopez R, et al: Clustal W and Clustal X version 2.0. Bioinformatics 2007, 23(21):2947-2948.

doi:10.1186/1472-6807-12-22

Cite this article as: Argiriadi et al.: Enabling structure-based drug design of Tyk2 through co-crystallization with a stabilizing aminoindazole inhibitor. BMC Structural Biology 2012 12:22. 